\title{
L'activité physique et le cancer: une étude transversale sur les facteurs de dissuasion et de facilitation face à l'exercice durant le traitement du cancer
}

par Stephanie Fernandez, Jenna Franklin, Nafeesa Amlani, Christian DeMilleVille, Dana Lawson et Jenna Smith

\section{RÉSUMÉ}

Cette enquête visait à explorer les facteurs de dissuasion et de facilitation face à l'exercice parmi des personnes atteintes de cancer en Ontario. Nous avons utilisé une enquête ponctuelle en ligne pour recueillir des données qualitatives et quantitatives. Nous avons formé un échantillon accidentel de personnes ayant un diagnostic actuel ou passé de n'importe quel type de cancer. Puis nous avons produit des pourcentages et des thèmes à partir des données.

Nous avons recueilli des données auprès de 30 personnes. Parmi elles, 63,3\% ont indiqué s'adonner à une activité physique peu fréquente et de faible intensité pendant le traitement. Les obstacles face à l'exercice durant le traitement comprenaient les symptômes physiques et le manque de connaissances au sujet des programmes d'exercice. Quant aux facteurs de facilitation, ils comprenaient l'accessibilité et l'expérience antérieure positive avec l'exercice. Plus de

\section{AU SUJET DES AUTEURS}

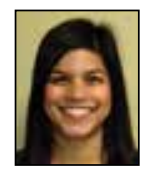

Stephanie Fernandez, M.Sc.(Physiothérapie), McMaster University School of Rehabilitation Sciences, Institute for Applied Health Sciences, Rm. 402, 1400 Main St. W, Hamilton, ON L8S $1 C 7$

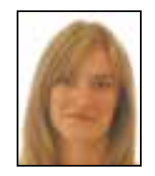

Jenna Franklin, M.Sc.(Physiothérapie), McMaster University School of Rehabilitation Sciences, Institute for Applied Health Sciences, Rm. 402, 1400 Main St. W, Hamilton, ON L8S 1C7

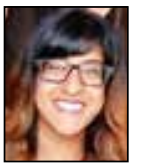

Nafeesa Amlani, M.Sc.(Physiothérapie), McMaster University School of Rehabilitation Sciences, Institute for Applied Health Sciences, Rm. 402, 1400 Main St. W, Hamilton, ON L8S 1C7

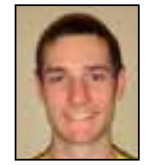

Christian DeMilleVille, M.Sc.(Physiothérapie), McMaster University School of Rehabilitation Sciences, Institute for Applied Health Sciences, Rm. 402, 1400 Main St. W, Hamilton, ON L8S 1 C7

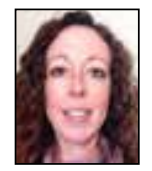

Dana Lawson, HBKin, Physio-Care Services, 845 Upper James Street \#208, Hamilton, ON L9C 3A3

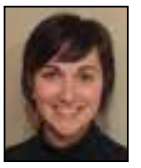

Jenna Smith, M.Sc.(Physiothérapie), McMaster University School of Rehabilitation Sciences, Institute for Applied Health Sciences, Rm. 402, 1400 Main St. W, Hamilton, ON L8S 1C7; PhysioCare Services, 845 Upper James Street \#208, Hamilton, ON L9C 3 A3

Auteure à qui adresser la correspondance : Jenna Smith, McMaster University School of Rehabilitation Sciences, Institute for Applied Health Sciences, Rm. 403, 1400 Main St. W, Hamilton, ON L8S 1C7. Courriel: jenna.smith@shaw.ca Tél. : 905-869-0599 ; Téléc. : 905-524-0069

DOI: $10.5737 / 2326880762514348$
$80 \%$ des participants n'avaient pas reçu d'information sur l'importance de l'exercice.

Les résultats de cette étude devraient encourager les professionnels de la santé qui travaillent en oncologie à éduquer les patients quant à l'importance de l'exercice.

Points clés

- Deux tiers des personnes atteintes de cancer réduisent leur niveau d'exercice durant le traitement

- Les principaux facteurs de dissuasion face à l'exercice pendant le traitement étaient la fatigue et le manque de connaissances sur les bienfaits de l'exercice et les programmes disponibles

- Il serait avantageux pour les patients atteints de cancer de recevoir des renseignements complets et cohérents sur l'exercice pendant le traitement du cancer.

\section{INTRODUCTION}

T e cancer est la cause première de décès au Canada (Société Lcanadienne du cancer, 20I3). La maladie et ses traitements devraient coûter au système de santé canadien quelque I77,5 milliards de $\$$ au cours des 30 prochaines années (Smetanin $\&$ Kobak, 2005). Le cancer et ses traitements entraînent plusieurs effets secondaires, dont une sensation persistante et subjective de fatigue physique, émotionnelle ou cognitive (Madden \& Newton, 2006). Les autres effets secondaires signalés comprennent la douleur, des effets cardiovasculaires, neurologiques, hormonaux et musculosquelettiques, ainsi que d'autres changements systémiques (Schmidtz et al., 20IO).

D'après la recherche, l'exercice atténue les divers effets secondaires du cancer et de ses traitements, peu importe le type ou le stade de la maladie (Velthuis, Agasi-Idenburg, Aufdemkampe \& Wittink, 20Iо; Knols, Aaronson, Uebelhart, Fransen \& Aufdemkampe, 2005). Selon une revue systématique Cochrane, l'exercice a d'importants effets positifs sur la qualité de vie liée à la santé parmi les patients atteints de cancer qui subissent un traitement actif, et les intensités d'exercice modérées à élevées produisent les effets les plus importants (Mishra et al., 20I2). De plus, différents types d'exercice produisent des effets positifs sur les mesures physiologiques - quel que soit le type de cancer-y compris une diminution de la douleur et de la nausée, ainsi qu'une augmentation de la force et de l'amplitude de mouvement des épaules (Knols et al., 2005). L'exercice a également des effets sur certains domaines de la santé mentale, par exemple la réduction de symptômes de dépression chez les survivants du cancer (Craft, Vanlterson, Helenowski, Rademaker \& Courneya, 20II). 
Malgré les bienfaits reconnus de l'exercice, seuls $22 \%$ des survivants du cancer au Canada faisaient de l'activité physique (AP), dont la définition correspond à environ une heure de marche par jour (Courneya, Katzmarzyk \& Bacon, 2008). La fatigue et la perte de forme physique, deux effets secondaires courants lors d'un traitement, sont des obstacles physiques pouvant mener à l'isolement social et au manque d'exercice routinier (Blaney et al., 20Io). De plus, Blaney et ses collaborateurs (20IO) ont observé que la perception d'obstacles environnementaux et financiers comme les programmes d'exercice non spécifiques et les coûts élevés peuvent contribuer à la baisse du niveau d'AP. Dans une récente étude transversale réalisée au Royaume-Uni, les principaux obstacles à l'exercice signalés par les participants étaient la rigidité des articulations, la fatigue et la douleur, soit trois facteurs qui peuvent être réduits par une participation régulière à des AP (Blaney, LoweStrong, Rankin-Watt, Campbell \& Gracey, 2013). Un autre obstacle cité était le manque de renseignements fournis sur le lien entre le cancer et l'exercice (Blaney et al., 2013). Dans la même optique, Blaney et ses collaborateurs (2013) ont montré que les facteurs suivants peuvent faciliter l'AP: un programme varié et agréable, l'approbation d'un professionnel de la santé ainsi que des objectifs et des interventions de traitement personnalisés. Cependant, on observe une variation dans la documentation scientifique, et il semble que les facteurs de facilitation particuliers dépendent du sexe des patients, du type de cancer et d'autres variables individuelles.

Bien que la documentation ait signalé de multiples bienfaits de l'exercice pour les personnes atteintes de cancer, peu de patients s'adonnent régulièrement à des AP (Cheifetz, 2013). Cela dit, la plupart de ces études étaient axées sur le cancer du sein ou ont été menées en dehors de l'Ontario (Canestraro et al., 2013). Cette étude visait à explorer les facteurs de dissuasion et de facilitation face à l'exercice parmi des personnes atteintes de cancer en Ontario qui recevaient ou avaient déjà reçu un traitement. Un deuxième objectif consistait à déterminer si ces facteurs de dissuasion et de facilitation variaient selon le type de cancer. Nous avons émis les hypothèses suivantes: (I) les obstacles comprendront la douleur, la fatigue et d'autres symptômes physiques comme la faiblesse ou la nausée, ainsi qu'un manque de connaissances au sujet des programmes existants et des bienfaits de l'exercice; (2) les facilitateurs comprendront la participation antérieure à des AP et l'éducation sur les programmes offerts et les bienfaits de l'exercice.

\section{MÉTHODOLOGIE}

\section{Conception et questionnaire}

Cette étude transversale a utilisé une enquête anonyme en ligne afin de recueillir des données quantitatives et qualitatives. L'enquête qui comprenait 23 questions a été administrée au moyen de SurveyMonkey. L'équipe de recherche s'est basée sur son expérience clinique et sur des études pertinentes pour créer le questionnaire. Nous avons obtenu au préalable le consentement éclairé des participants à l'enquête. Ces derniers remplissaient le questionnaire à domicile, dans la clinique des chercheuses principales ou dans un lieu de leur choix. Le questionnaire prenait Io-20 minutes à remplir. Les questions portaient sur la fréquence et l'intensité de l'exercice avant le diagnostic, ainsi que pendant et après le traitement contre le cancer. Nous avons également étudié les obstacles et facilitateurs perçus face à l'exercice, ainsi que la fourniture, par les prestataires de soins, de renseignements liés à l'exercice. Aux fins de cette étude, les termes exercice et AP étaient synonymes. Nous avons obtenu l'approbation du comité de déontologie de McMaster en février 20I3.

\section{Participants}

Nous avons recruté un échantillon accidentel de personnes atteintes de cancer qui participaient à un programme de physiothérapie en clinique externe ou à des activités établies de soutien aux personnes atteintes de cancer dans différentes collectivités ontariennes. Les individus qui répondaient aux critères d'inclusion vivaient en Ontario, avaient plus de I8 ans, avaient un diagnostic actuel ou passé d'un cancer de n'importe quel type et recevaient présentement—ou avaient reçu —un traitement contre le cancer. On ne stipulait aucune période maximale écoulée depuis la fin du traitement. Nous avons demandé à tous les participants de se souvenir de leur comportement durant le traitement. Les participants ont été trouvés par leurs professionnels de la santé, qui leur ont demandé de participer à l'étude.

\section{Collecte de données et analyse}

Les données ont été recueillies au cours d'une période de trois mois, puis soumises à une analyse quantitative et à une méthode qualitative additionnelle. Les questions fermées ont fait l'objet d'une analyse quantitative qui a permis de calculer des pourcentages. Les réponses aux questions ouvertes ont été interprétées au moyen d'une analyse qualitative du contenu. Cela implique le recensement et la codification de l'information en catégories distinctes, ce qui permet ensuite de dégager des thèmes (Hsieh \& Shannon, 2005). Cette interprétation est de nature subjective, et aucune notion préconçue concernant les thèmes n'est imposée (Hsieh \& Shannon, 2005). Deux chercheuses (JF et $\mathrm{SF}$ ) ont indépendamment révisé, interprété et organisé les données en thèmes. Elles ont discuté des résultats entre elles, puis avec la chercheuse principale, jusqu'à l'atteinte d'un consensus. Les participants n'étaient pas tenus de répondre à toutes les questions, mais seuls les questionnaires remplis à au moins $85 \%$ ont été retenus pour l'étude. Nous avons examiné les réponses offertes à chaque question par l'ensemble de l'échantillon, puis analysé les réponses individuelles.

\section{RÉSULTATS}

\section{Caractéristiques des participants}

$\mathrm{Au}$ total, 30 personnes ayant un diagnostic de cancer actuel ou passé ont pris part à l'enquête. Le tableau I contient des renseignements démographiques détaillés sur les participants. Vingt-huit des participants étaient des femmes (93,I\%), et l'âge moyen au moment du diagnostic était de 47,8 ans (é.-t. $=+/-15,8)$. Le diagnostic le plus courant était le cancer du sein $(58,6 \%)$, et les stades les plus courants étaient le stade I $(63,2 \%)$ et le stade II $(2 \mathrm{I}, 0 \%)$. La quasi-totalité des participants 
Tableau 1: Données démographiques des participants

\begin{tabular}{|c|c|c|c|c|c|c|c|c|c|c|c|c|}
\hline \multirow{2}{*}{$\begin{array}{l}\text { Données } \\
\text { démographiques }\end{array}$} & \multirow{2}{*}{\begin{tabular}{|l} 
Sexe \\
Féminin
\end{tabular}} & \multicolumn{2}{|l|}{ Âge } & \multicolumn{2}{|c|}{ Type de cancer } & \multicolumn{4}{|c|}{ Stade du cancer } & \multicolumn{3}{|l|}{ Traitement reçu } \\
\hline & & $\leq 50$ & $>50$ & Sein & Autre & 1 & 2 & 3 & 4 & Chimiothérapie & Radiothérapie & Chirurgie \\
\hline N & 27 & 15 & 16 & 17 & 12 & 12 & 4 & 1 & 2 & 21 & 14 & 23 \\
\hline$\%$ & 93,1 & 48,3 & 51,7 & 58,6 & 41,4 & 63,2 & 21 & 5,3 & 10,5 & 72,4 & 48,3 & 79,3 \\
\hline
\end{tabular}

Tableau 2: Niveau d'activité physique des participants

\begin{tabular}{|l|l|l|l|l|l|l|l|l|l|l|l|l|l|l|l|}
\hline $\begin{array}{l}\text { Niveau } \\
\text { d'activité }\end{array}$ & \multicolumn{2}{|l|}{ Fréquence } & \multicolumn{1}{l|}{ Intensité } \\
\cline { 2 - 22 } & Fréquent & Modéré & Occasionnel & Peu fréquent & 0 & 1 & 2 & 3 & 4 & 5 & 6 & 7 & 8 & 9 & 10 \\
\hline
\end{tabular}

Avant le diagnostic de cancer

\begin{tabular}{|l|c|c|c|c|c|c|c|c|c|c|c|c|c|c|c|}
\hline $\mathrm{N}$ & 2 & 11 & 12 & 5 & 3 & 1 & 6 & 0 & 2 & 11 & 3 & 2 & 2 & 0 & 0 \\
\hline$\%$ & 6,7 & 36,7 & 40 & 16,7 & 10 & 3,3 & 20 & 0 & 6,7 & 36,7 & 10 & 6,7 & 6,7 & 0 & 0 \\
\hline
\end{tabular}

Durant le traitement contre le cancer

\begin{tabular}{|l|c|c|c|c|c|c|c|c|c|c|c|c|c|c|l|}
\hline$N$ & 0 & 6 & 5 & 19 & 19 & 0 & 4 & 1 & 1 & 5 & 0 & 0 & 0 & 0 & 0 \\
\hline$\%$ & 0 & 20 & 16,7 & 63,3 & 63,3 & 0 & 13,3 & 3,3 & 3,3 & 16,7 & 0 & 0 & 0 & 0 & 0 \\
\hline
\end{tabular}

Après le traitement contre le cancer

\begin{tabular}{|l|c|c|c|c|c|c|c|c|c|c|c|c|c|c|c|}
\hline $\mathrm{N}$ & 3 & 12 & 10 & 3 & 1 & 1 & 8 & 0 & 1 & 10 & 2 & 1 & 4 & 0 & 0 \\
\hline$\%$ & 10,7 & 42,9 & 35,7 & 10,7 & 3,6 & 3,6 & 28,6 & 0 & 3,6 & 35,7 & 7,1 & 3,6 & 14,3 & 0 & 0 \\
\hline
\end{tabular}

*Niveaux de fréquence: Fréquent (6-7 fois/semaine), Modéré (3-5 fois/semaine), Occasionnel (1-2 fois /semaine), Peu fréquent ( $<1$ fois / semaine)

**Niveaux d'intensité: 0 (intensité très peu élevée [p. ex. marche très lente]), 2 (intensité peu élevée [p. ex. marche normale]), 5 (intensité modérée [p. ex. marche rapide, sortie en vélo ou activité provoquant la transpiration d'ici la fin]), 8 (intensité élevée [p. ex. course à pied, fréquence cardiaque accrue, transpiration et élévation de la fréquence respiratoire]), 10 (intensité très élevée [p. ex. entraînement au marathon]).

(96,7\%) ont indiqué avoir reçu un traitement lié au cancer, le plus fréquent étant la chirurgie $(79,3 \%)$, devant la chimiothérapie $(72,4 \%)$.

\section{Niveau d'activité physique}

On a interrogé les participants quant à leur niveau d'AP avant, pendant et après leur traitement contre le cancer (voir le tableau 2). Avant le diagnostic de cancer, la plupart des participants indiquaient une fréquence d'AP occasionnelle (40\%, I-2 fois/semaine) ou modérée (36,7\%, 3-5 fois/semaine). L'AP la plus fréquente $(36,7 \%)$ était celle d'intensité modérée, définie comme une activité à rythme soutenu qui provoque la transpiration.

Durant le traitement, la plupart des participants $(63,3 \%)$ ont signalé s'être adonnés de façon peu fréquente $(<$ I fois $/$ semaine) à une AP d'intensité très peu élevée, soit l'équivalent d'une marche lente. Peu d'individus ( $16,7 \%)$ ont maintenu un degré d'activité modéré pendant le traitement. Les personnes atteintes d'un cancer du sein étaient les plus nombreuses dans ce groupe $(23,5 \%)$, alors que les participants atteints d'un autre cancer affichaient un taux d'AP modérée de i6,7\%.

Après le traitement, les types d'AP les plus courants étaient ceux de fréquence modérée $(42,9 \%)$ et occasionnelle $(35,7 \%)$.
L'intensité signalée par la majorité des participants était modérée $(35,7 \%)$. Lorsqu'on a demandé aux participants ce qui avait motivé un changement dans leur niveau d'AP, les réponses les plus courantes étaient que les participants se sentaient mieux $(65,4 \%)$ et avaient plus d'énergie $(61,5 \%)$. Après le traitement, les personnes atteintes d'un cancer du sein ont augmenté leur niveau d'AP plus que les autres participants ( $92,8 \%$ contre $80 \%)$.

\section{Commentaires écrits}

Nous avons posé des questions ouvertes sur le type d'AP auquel les participants s'adonnaient avant leur diagnostic et pendant leur traitement. Les types communs d'exercice avant le diagnostic comprenaient les sports d'endurance, la musculation, différentes activités récréatives et les cours d'exercice organisés. (Voir la figure I pour une représentation visuelle de ces thèmes.) Les sports d'endurance étaient les plus courants et comprenaient la marche, la natation, le vélo et la course. Les activités récréatives, les cours d'exercice organisés et la musculation étaient également signalés.

Durant le traitement, l'activité d'endurance demeurait la plus populaire. La plupart des participants ont indiqué que leur activité préférée était la marche. Les participants ont 


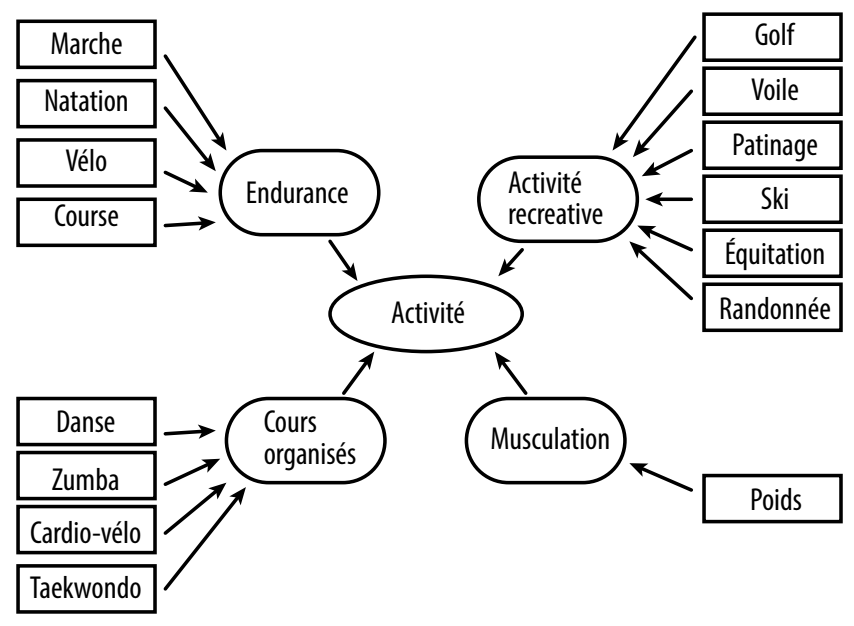

Figure $\mathrm{I}$ : Thèmes courants parmi les types d' exercice avant le diagnostic de cancer

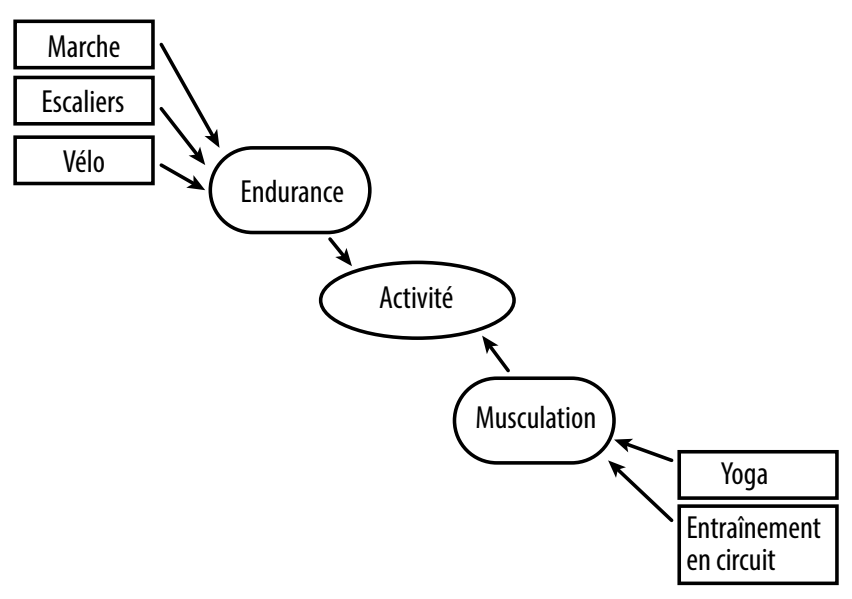

Figure 2: Thèmes courants parmi les types d'exercice durant le traitement contre le cancer

Tableau 3 : Obstacles à la participation à des AP

\begin{tabular}{|l|c|c|c|c|c|c|c|}
\hline Obstacles & $\begin{array}{c}\text { Manque } \\
\text { d'énergie }\end{array}$ & $\begin{array}{c}\text { Symptômes } \\
\text { physiques }\end{array}$ & $\begin{array}{c}\text { Ignorance des } \\
\text { programmes }\end{array}$ & $\begin{array}{c}\text { Défis } \\
\text { physiques }\end{array}$ & $\begin{array}{c}\text { Mauvaises perceptions } \\
\text { de l'exercice }\end{array}$ & $\begin{array}{c}\text { Ignorance de la nécessité de } \\
\text { faire de l'exercice }\end{array}$ & $\begin{array}{c}\text { Autres } \\
\text { N }\end{array}$ \\
\hline 2 & 20 & 14 & 10 & 7 & 6 \\
\hline$\%$ & 81,5 & 74,1 & 51,9 & 37 & 25,9 & 22,2 \\
\hline
\end{tabular}

également pris part à des cours d'exercice organisés pendant leur traitement, dont des cours de yoga et des programmes offerts dans des centres comme Wellspring et le YMCA (voir la figure 2).

\section{Facteurs de dissuasion face à l'exercice et effets secondaires des traitements}

On a questionné les participants concernant les obstacles face à l'exercice pendant leur traitement contre le cancer. Les trois réponses principales étaient le manque d'énergie (8I,5\%), les symptômes physiques (74,I\%) et l'ignorance des programmes offerts (5I,9\%). Les autres obstacles comprenaient les défis physiques, la croyance qu'ils se sentiraient encore moins bien en faisant de l'exercice et l'ignorance du fait qu'ils devraient faire de l'exercice (voir le tableau 3). Lors de la comparaison des résultats selon le type de cancer, les personnes ayant reçu un diagnostic de cancer du sein signalaient des niveaux d'énergie moins élevés que les autres participants $(82,4 \%$ contre $66,7 \%)$, mais elles étaient mieux informées que les autres au sujet des programmes d'exercice offerts $(50 \%$ contre 4I,2\%).

Les trois effets secondaires les plus courants en lien avec les traitements étaient la fatigue $(89,7 \%)$, l'engourdissement et/ou les picotements dans les extrémités (69\%) et la faiblesse musculaire $(65,5 \%)$. Les autres effets secondaires comprenaient la nausée $(51,7 \%)$, la douleur $(48,3 \%)$, la dépression (48,3\%), l'anxiété (4I,4\%), les mouvements limités au niveau des articulations $(27,6 \%)$ et les vomissements $(24, \mathrm{I} \%)$. La plupart des participants $(76,7 \%)$ ont indiqué que ces symptômes physiques influençaient leur capacité ou leur volonté de faire de l'exercice. Lors de la comparaison des résultats selon le type de cancer, les personnes diagnostiquées d'un cancer du sein signalaient des niveaux plus élevés de faiblesse musculaire $(70,6 \%)$ et moins d'engourdissement et de picotements dans les extrémités $(58,8 \%)$ que celles des autres groupes.

Facteurs de facilitation face à l'exercice pendant le traitement

Les trois facteurs facilitants durant le traitement étaient l'expérience antérieure positive avec l'exercice $(63,2 \%)$, un désir de poursuivre une routine d'exercice adoptée avant le début du traitement $(47,4 \%)$ et la facilité d'accès à des services d'exercice $(42, \mathrm{I} \%)$. D'autres facteurs de facilitation comprenaient: les participants avaient entendu dire que l'exercice était bon pour eux; les participants avaient des proches avec qui faire de l'exercice; et les participants avaient plus de temps pour faire de l'exercice (voir le tableau 4). Lors de la comparaison des résultats selon le type de cancer, le fait d'avoir une expérience passée positive avec l'exercice était un facteur facilitant beaucoup plus fort parmi les personnes atteintes de cancer du sein (75\%) que parmi celles des autres groupes $(25 \%)$.

\section{ÉDUCATION}

Nous avons également interrogé les participants quant à la présence et au type d'information fournie par les professionnels de la santé en lien avec l'AP durant le traitement du cancer (voir le tableau 5). Les personnes atteintes de cancer du sein étaient plus conscientes de l'importance de l'exercice que les autres groupes (58,8\% contre 33,3\%). Au total, 83,3\% des 
Tableau 4 : Facteurs facilitants de la participation à des AP

\begin{tabular}{|l|l|l|l|l|l|l|}
\hline $\begin{array}{l}\text { Facteurs } \\
\text { facilitants }\end{array}$ & $\begin{array}{l}\text { Expérience positive } \\
\text { préalable sur } \\
\text { l'exercice }\end{array}$ & $\begin{array}{l}\text { Maintenir les } \\
\text { niveaux de condition } \\
\text { ph. }\end{array}$ & $\begin{array}{l}\text { Programme } \\
\text { d'exercice accessible }\end{array}$ & $\begin{array}{l}\text { Perceptions positives } \\
\text { de l'exercice }\end{array}$ & $\begin{array}{l}\text { Encouragement } \\
\text { d'êtres chers }\end{array}$ & $\begin{array}{l}\text { Temps } \\
\text { libre }\end{array}$ \\
\hline $\mathrm{N}$ & 12 & 9 & 8 & 7 & 6 & 5 \\
\hline$\%$ & 63,2 & 47,4 & 42,1 & 36,8 & 9 & 31,6 \\
\hline
\end{tabular}

Tableau 5 : Éducation liée à l'activité physique durant le traitement anticancéreux

\begin{tabular}{|l|l|l|l|}
\hline $\begin{array}{l}\text { Éducation } \\
\text { dispensée par: }\end{array}$ & l'oncologue & $\begin{array}{l}\text { l'infirmière de } \\
\text { soins intégraux }\end{array}$ & $\begin{array}{l}\text { n'importe quel } \\
\text { professionnel }\end{array}$ \\
\hline Oui & 5 & 4 & 12 \\
\hline Non & 25 & 26 & 18 \\
\hline
\end{tabular}

oncologues et $86,7 \%$ des infirmières n'enseignaient pas aux patients l'importance de l'exercice durant leur traitement du cancer. De plus, $66 \%$ ont indiqué que personne ne leur avait fourni une quelconque instruction ou information générale sur l'AP pendant le traitement.

\section{DISCUSSION}

Les résultats de cette étude démontrent l'influence du cancer et des traitements du cancer sur la capacité des patients à faire de l'exercice, ainsi que l'importance de l'éducation (sur l'AP) pour promouvoir l'exercice au sein de cette population. Avant le diagnostic, I7\% des participants s'adonnaient à des AP peu fréquentes. Ce pourcentage est passé à $66,7 \%$ durant le traitement. Sur le plan individuel, les deux tiers des participants sont passés au niveau inférieur de fréquence d'AP (p. ex. de fréquent à modéré, ou de modéré à peu fréquent) pendant le traitement. Après le traitement, on a observé que la plupart des patients, quel que soit leur type de cancer, retrouvaient leur niveau d'activité prédiagnostique.

Les données recueillies dans le cadre de cette étude appuient la recherche passée qui démontre que les obstacles courants face à l'exercice pendant le traitement comprennent la fatigue et le manque de connaissances sur les programmes existants et sur les bienfaits de l'exercice. De plus, les effets secondaires physiques des traitements avaient une influence négative sur les niveaux d'activité, tous types de cancer confondus. Ce constat correspond aux résultats d'autres études en ce qui concerne les facteurs de dissuasion face à l'exercice (Sander, Wilson, Izzo, Mountford \& Hayes, 20I2; Brunet, Taran, Burke \& Sabiston, 2013).

Plus de la moitié des participants à notre étude ont indiqué ne pas être conscients des bienfaits de l'exercice pour la gestion des symptômes du cancer. Quatre personnes en particulier ont signalé avoir reçu de l'information sur l'AP, mais n'étaient pas au courant des effets positifs de l'exercice. Ce manque d'information complète est un thème commun dans la documentation scientifique (Cheifetz, 2013; Ottenbacher et al., 20II). Dans une étude quantitative, Sander et ses collègues
(2012) ont rapporté que les personnes atteintes de cancer du sein signalaient avoir peur de faire de l'exercice, car elles ne savaient pas quels exercices étaient sécuritaires pour elles. Nos résultats illustrent donc l'importance d'offrir une éducation complète et cohérente aux personnes atteintes d'un cancer pour réduire l'appréhension face à l'exercice. Finalement, les répondants de notre étude n'étaient pas au courant des programmes d'exercice offerts. Les professionnels de la santé devraient donc penser à inclure de l'information sur les programmes d'exercice et les ressources communautaires dans les conseils qu'ils dispensent régulièrement (Jones \& Courneya, 2002).

Cette étude permet en outre de tirer de nouvelles conclusions lorsqu'on compare les facteurs de dissuasion et de facilitation face à l'exercice en fonction du type de cancer. Notamment, les personnes atteintes de cancer du sein signalaient des niveaux de fatigue et de faiblesse musculaire plus élevés que celles des autres groupes. Ce résultat n'est pas surprenant, puisque les traitements et les médicaments utilisés en chimiothérapie diffèrent selon le type de cancer et entraînent des effets secondaires différents. Autre résultat important: les personnes atteintes d'un cancer du sein recevaient plus souvent de l'information-de la part d'un professionnel de la santé — au sujet de l'importance de l'AP durant le traitement et étaient donc plus conscientes de la nécessité de faire de l'exercice pendant le traitement. Ce résultat est possiblement attribuable au fait qu'il existe plus de documentation sur les bienfaits de l'exercice pour les personnes atteintes de cancer du sein que sur les bienfaits de l'exercice pour les autres types de cancer. Les protocoles de traitement pour différents types de cancer ont peut-être également un rôle à jouer dans ce résultat, puisque les personnes atteintes d'un cancer $\mathrm{du}$ sein sont souvent prises en charge par une équipe pluridisciplinaire dès le début de leur traitement.

\section{Implications cliniques}

Les résultats de cette étude ont des implications pour l'intégration de programmes d'exercice destinés aux personnes qui reçoivent un traitement contre le cancer. Nommément, les résultats illustrent l'importance de l'éducation sur l'AP et de la réduction des obstacles liés aux symptômes physiques tôt dans le traitement. Bon nombre de professionnels de la santé (p. ex. physiothérapeutes et infirmières) possèdent suffisamment d'information pour recommander des exercices et offrir une éducation sur ce sujet. Cependant, leurs services sont actuellement sous-utilisés dans le contexte de la réadaptation liée au cancer (Cheifetz, 20I3). 


\section{LIMITES}

Il est important de tenir compte des limites de cette étude lorsqu'on en considère les résultats. Tout d'abord, notre échantillon était petit et principalement féminin, ce qui complique la généralisation des résultats à une plus grande population. De plus, la présence de facteurs de comorbidité inconnus ou de variables confusionnelles au sein de cette population a possiblement biaisé les résultats. Finalement, les données incluses dans cette étude ont été recueillies de façon rétrospective, ce qui introduit possiblement un biais de rappel.

\section{CONCLUSION}

Cette étude a confirmé que les niveaux d'exercice diminuent lorsque les patients subissent un traitement anticancéreux. Comme nous l'avions supposé, la fatigue, d'autres symptômes physiques et le manque de connaissances concernant l'exercice et ses bienfaits étaient les facteurs de dissuasion les plus courants face à l'exercice. La douleur était un facteur important.

\section{RÉFÉRENCES}

Blaney, J.M., Lowe-Strong, A., Rankin, J., Campbell, A., Allen, J., \& Gracey, J. (20I0). The cancer rehabilitation journey: Barriers to and facilitators of exercise among patients with cancer-related fatigue. Physical Therapy, 90, II35-47.

Blaney, J.M., Lowe-Strong, A., Rankin-Watt, J., Campbell, A., \& Gracey, J.H. (2013). Cancer survivors' exercise barriers, facilitators and preferences in the context of fatigue, quality of life and physical activity participation: A questionnaire-survey. Psychooncology, 22, I86-94.

Brunet, J., Taran, S., Burke, S., \& Sabiston, C.M. (2013). A qualitative exploration of barriers and motivators to physical activity participation in women treated for breast cancer. Disability and Rehabilitation, 24, 2038-2045.

Canestraro, A., Nakhle, A., Stack, M., Strong, K., Wright, A., Beauchamp, M., et al. (2013). Oncology rehabilitation provision and practice patterns across Canada. Physiotherapy Canada, 65, 94-IO2.

Cheifetz, O. (2013). Clinicians commentary on Sing et al. Physiotherapy Canada, 65, 192-3.

Courneya, KS., Katzmarzyk, PT. \& Bacon, E. (2008). Physical activity and obesity in Canadian cancer survivors. Cancer, 112, 2475-82.

Craft, L.L., Vanlterson, E.H., Helenowski, I.B., Rademaker, A.W., \& Courneya, K.S. (20II). Exercise effects on depressive symptoms in cancer survivors: A systematic review and meta-analysis. Cancer Epidemiology, Biomarkers and Prevention, 21, 3-19.

Hsieh, H.F., \& Shannon, S.E. (2005). Three approaches to qualitative content analysis. Qualitative Health Research, 15, I277-88.

Jones, L.W., \& Courneya, K.S. (2002). Exercise counseling and programming preferences of cancer survivors. Cancer Practice, 10, 208-I5.

Knols, R., Aaronson, N.K., Uebelhart, D., Fransen, J., \& Aufdemkampe, G. (2005). Physical exercise in cancer patients during and after medical treatment: A systematic review of randomized and controlled clinical trials. Journal of Clinical Oncolog $\gamma, 23,3830-3842$.
Nous avons confirmé notre hypothèse quant au rôle d'un engagement antérieur avec l'exercice. Les autres facteurs de facilitation étaient l'expérience antérieure positive avec l'exercice et l'accès à des services d'exercice. De plus, nos résultats correspondaient à la documentation disponible sur ce sujet et confirmaient l'importance du rôle des professionnels de la santé dans la réadaptation liée au cancer. Les différences observées dans les résultats entre le cancer du sein et les autres types de cancer étaient intéressantes. Les personnes atteintes d'un cancer du sein ont signalé des degrés plus élevés de fatigue et de faiblesse musculaire, ainsi que d'éducation et de connaissances concernant l'importance de l'exercice durant le traitement anticancéreux.

\section{REMERCIEMENTS}

Les auteurs souhaitent remercier le réseau Wellspring (un réseau de soutien aux personnes atteintes de cancer) et le programme Can Well pour leur assistance dans le recrutement des participants.

Madden, J., \& Newton, S. (2006). Why am I so tired all the time? Understanding cancer-related fatigue. Clinical Journal of Oncology Nursing, 10(5), 659-66I.

Mishra, S.I., Scherer, R.W., Geigle, P.M., Berlanstein, D.R., Topaloglu, O., Gotay, C.C., et al. (2012). Exercise interventions on healthrelated quality of life for cancer survivors. Cochrane Database of Systematic Reviews, 8, CDoo7566.

Ottenbacher, A.J., Day, R.S., Taylor, W.C., Sharma, S.V., Sloane, R., Snyder, D.C., et al. (20II). Exercise among breast and prostate cancer survivors-What are their barriers? Journal of Cancer Survivorship, 5.4, 4I3-I9.

Sander, A.P., Wilson, J., Izzo, N., Mountford, S.A., \& Hayes, K.W. (2OI2). Factors that affect decisions about physical activity and exercise in survivors of breast cancer: A qualitative study. Physical Therapy, 92, 525-36.

Schmitz, K.H., Courneya, K.S., Matthews, C., Demark-Wahnefried, W., Galvao, D.A., Pinto, B.M, et al. (20I0). American College of Sports Medicine roundtable on exercise guidelines for cancer survivors. Medicine \& Science in Sports Q Exercise, 42(7), I409-I426.

Smetanin, P., \& Kobak, P. (2005). Interdisciplinary Cancer Risk Management: Canadian Life and Economic Impacts. Risk Analytica.

Société canadienne du cancer, Comité directeur sur les statistiques sur le cancer. (2013). Statistiques canadiennes sur le cancer 2013. Récupéré à: http://www.cancer.ca/ /media/cancer.ca/CW/ cancer\%2oinformation/cancer\%20I0I/Canadian\%2ocancer\%20 statistics/canadian-cancer-statistics-2013-FR.pdf

Velthuis, M.J., Agasi-Idenburg, S.C., Aufdemkampe, G., \& Wittink, H.M. (2010). The effect of physical exercise on cancer-related fatigue during cancer treatment: A meta-analysis of randomised controlled trials. Clinical Oncology, 22(3), 208-22I. 